\title{
La ficha cualitativa como herramienta etnográfica para evidenciar el favorecimiento del desarrollo del pensamiento tecnológico de niños y niñas*
}

\author{
Qualitative data as a ethnographic tool to prove \\ the enhancement of children technological thinking
}

Artículo recibido: febrero de 2011

Artículo aprobado: mayo de 2011
Diana Luz López Ortega Martha Betancur Taborda Olga Palacio $N$. $^{* * * *}$

Orlando Torres $M$.

\section{Resumen}

Este artículo presenta algunos resultados obtenidos a partir de la sistematización de los insumos de la vivencia escolar de IV semestre (práctica docente), durante los años 2003 (primer semestre), al 2006 (segundo semestre), en la Licenciatura de Pedagogía Infantil de la Facultad de Ciencias y Educación de la Universidad Distrital Francisco José de Caldas, Bogotá, Colombia. El propósito de la presente investigación, es aproximarse o lograr que los estudiantes de cuarto semestre capten el significado de los conceptos «artefacto cultural»y «pensar tecnológicamente» para que puedan dotar de sentido la actividad pedagógica que realizan con los niños de 4 a 6 años promedio. Se hace énfasis en la ficha cualitativa como herramienta etnográfica, donde el resultado de ésta es una propuesta metodológica estructurada y afinada para el aula universitaria.

\section{Palabras clave:}

Desarrollo del pensamiento tecnológico, ficha cualitativa, artefacto cultural, formación de docentes, ciencia y tecnología.

Este proyecto de innovación en el aula fue financiado, parcialmente, por El Centro de Investigaciones y Desarrollo Científico CIDC, de la universidad Distrital Francisco José de Caldas (2006-2008).

* Licenciada en Física. Especialista en Ingeniería de Software. Magíster en Teleinformática. Profesora Hora Cátedra de la Facultad de Ciencias y Educación, Licenciatura en Pedagogía Infantil, Universidad Distrital Francisco José de Caldas. Grupo de investigación GALATEA. Correo electrónico: dlopez@udistrital.edu.co

*** Licenciada en Biología. Especialista en Educación Ambiental. Candidata a Magíster en Didáctica de las Ciencias. Profesora Hora Cátedra de la Facultad de Ciencias y Educación, Licenciatura en Pedagogía Infantil, Universidad Distrital Francisco José de Caldas. Grupo de investigación GALATEA. Correo electrónico: macebeta@hotmail.com

**** Antropóloga. Profesora Hora Cátedra de la Facultad de Ciencias y Educación, Licenciatura en Pedagogía Infantil, Universidad Distrital Francisco José de Caldas. Grupo de investigación GALATEA. Correo electrónico: ojpalacion@udistrital.edu.co

$* * * * *$ Licenciado en Electrónica y Electricidad. Profesor Hora Cátedra de la Facultad de Ciencias y Educación, Licenciatura en Pedagogía Infantil, Universidad Distrital Francisco José de Caldas. Grupo de investigación GALATEA. Correo electrónico: otorresm@udistrital.edu.co 


\section{Abstract}

This article presents some results from the systematization of the inputs of the school experience in IV semester (teaching practice) through the years 2003 - I / 2006 - II, in the Bachelor of Children's Pedagogy at the Science and Education Faculty in the Universidad Distrital "Francisco José de Caldas" Bogotá - Colombia. The purpose of this is for the fourth semester students to understand the meaning of the concepts of cultural device - to think technologically, and provide meaning to the pedagogical practices that they perform with children from 4 to 6 year old on average. The emphasis is placed on the qualitative data as ethnographic tool; the result is a methodological proposal, structured and made suitable for university students.

\section{Keywords}

Technological thinking development, qualitative data, cultural devices, teacher training, science and technology.

\section{Introducción}

En el plan de estudios del proyecto curricular de pedagogía infantil ${ }^{1}$, las vivencias escolares (práctica docente) estructuran horizontalmente el currículo en cada semestre, y, a su vez, éste articula un eje (plan de estudios correspondiente hasta el 2009 - I).

Para el caso que nos ocupa, la vivencia escolar ${ }^{2}$ de cuarto semestre, el eje articulador es el pensamiento tecnológico que pedagógicamente implica la relación adulto-niño. Dado el vacío conceptual, metodológico y didáctico, encontrado en este eje, el proyecto de innovación en el aula ${ }^{3}$, pretende ser una alternativa que involucre los resultados obtenidos durante el desarrollo del espacio académico, en mención, en los años correspondientes al 2003 (primer semestre) hasta el 2006 (segundo semestre).

1 Facultad de Ciencias y Educación, Universidad Distrital Francisco José de Caldas, Bogotá-Colombia.

2 Práctica docente.

3 Formación pedagógica para favorecer el pensamiento tecnológico de niños y niñas de 4 a 6 años promedio.
Para este trabajo de innovación se hizo una sistematización de los datos (informes cualitativos ${ }^{4} \mathrm{del}$ trabajo de campo con los niños), donde el resultado de ésta, es una propuesta metodológica estructurada y afinada para el aula universitaria con base en los resultados obtenidos de la misma ${ }^{5}$, es decir, la ficha cualitativa como registro etnográfico.

El presente artículo da cuenta solamente de los antecedentes, la definición del problema de innovación, la metodología, y, por último, de algunas conclusiones respecto a lo encontrado en el proceso de sistematización de esta vivencia.

\section{Antecedentes y referentes conceptuales}

Para contextualizar el problema de innovación se hace necesario un poco de historia. Ésta se trabajó inicialmente en el grupo de investigación INTERCITEC $^{6}$, a lo largo de varios años y en dos etapas, como se muestra a continuación.

Primera etapa: relaciones con el pasado. Se elaboraron unas definiciones iniciales, relacionadas con el área de la ciencia (hasta el 2006-II) que ya había marcado algunas diferencias con el empirismo y el positivismo, y cómo estas perspectivas afectaban la enseñanza de las ciencias; sin embargo, las implementaciones prácticas no lograron consolidar una alternativa concomitante con todos estos replanteamientos. Fueron varias las dificultades encontradas en esta etapa: a) las actividades se concentraban en la enseñanza de la ciencia y matemáticas escolares; no existía en el grupo una claridad sobre qué era la tecnología y mucho menos su papel en el ámbito educativo y en la educación infantil; b) la imagen reduccionista de lo que es la educación en este campo, encontrada en las instituciones, "[...] los objetos son para que los niños y niñas jueguen y se diviertan", que en sí misma no es una intención de poca trascendencia, pero que no revela la existencia de finalidades y propósitos más pedagógicos, sino que es similar a la forma como actúan los

4 O ficha cualitativa, la cual es un instrumento donde se registra el trabajo de campo (vivencia), y que da cuenta del proceso vivido con los niños en términos de la interacción entre la situación problema y artefacto.

5 Sistematización.

6 Hasta el 2006 - II. 
padres sin necesidad de ir a la universidad; c) que a pesar de todos los esfuerzos de formación realizados en el espacio universitario, con estudiantes de cuarto semestre, no se lograba una apropiación conceptual que les permitiera resolver el problema en la práctica; los niños seguían jugando muy divertidos, lo que satisfacía a los estudiantes, pero no se podía "observar aspectos cognitivos para el favorecimiento del pensamiento tecnológico" con las actividades que desarrollaban; d) la consecución de los recursos elaborados para tal fin se tornó en una gran dificultad.

Segunda etapa: surgimiento de un proyecto educativo en el ámbito tecnológico. El equipo ${ }^{8}$ se constituye como grupo de investigación con el propósito de trabajar en torno a la educación en ciencia y tecnología, con un enfoque cultural. A nivel conceptual, se logró una aproximación al pensamiento tecnológico como una forma de pensar tecnológicamente en un contexto cultural dado; es así como, en este marco, se prevé la acción pedagógica como una mediación con artefactos (Cole, 1999) $)^{9}$ que favorece este pensar. A pesar de los avances, la pregunta sigue siendo (aún en el 2011 - I) ¿cómo lograr que los estudiantes de cuarto semestre capten el significado de los conceptos («artefacto» y "pensar tecnológicamente») y doten de sentido la actividad pedagógica que ellos realizan con los niños?

\section{Definición y descripción del problema}

La contextualización, hecha con los antecedentes, implica captar el significado de lo que es un artefacto cultural, artefactos que median con lo tecnológico, aproximaciones no consumistas a procesos tecnológicos, objetos tecnológicos y situaciones que requieren soluciones tecnológicas, presentes en el entorno cotidiano del ciudadano. Por tal ra-

7 De la universidad.

8 Se refiere al área de la ciencia que como grupo de investigación incluía a los profesores que tenían a cargo esta asignatura (dos de ciencias y uno del área de sociedad). Este equipo funcionó hasta el año 2006-II.

9 Según Cole, los "artefactos cumplen la función básica de coordinar a los seres humanos con el mundo físico y entre sí. Son al mismo tiempo materiales e ideales (es decir, conceptuales o simbólicos), por lo que a la vez que permiten actuar sobre el mundo funcionan también significativamente" (1999). zón, toma sentido mantener vigente la pregunta: ¿cómo lograr que los estudiantes de cuarto semestre capten el significado de los conceptos («artefacto» $\mathrm{y}$ «pensar tecnológicamente») y doten de sentido la actividad pedagógica que ellos realizan con los niños?, ya que ella constituye el problema de innovación.

Así mismo, lo anterior requiere de un análisis social y cultural del fenómeno tecnológico, puesto que a partir de este contexto se puede comprender mejor lo que es un artefacto cultural. Sin embargo, la segunda parte de nuestra pregunta, implica que para el ámbito pedagógico podemos construir artefactos culturales que medien la acción educativa. Inicialmente, se trata de reconocer que los artefactos sirven para entender lo tecnológico, es decir, cómo ellos se constituyen en medios para realizar una acción: el favorecimiento del pensamiento tecnológico de los niños.

En virtud de lo anterior y para desarrollar el problema, se discriminan dos grandes procesos: la comprensión y la acción pedagógica. De un lado, la comprensión o captación del significado de los conceptos básicos que orientan la vivencia son la tecnología como un fenómeno profundamente social y cultural y, por lo tanto, con implicaciones políticas, económicas, cognitivas y religiosas para la vida social del hombre; de otra parte, la tecnología como una forma de imaginar y explicar el mundo con unas lógicas determinadas próximas al pensamiento paradigmático (Bruner, 1998: 23); el conocimiento y la exploración de artefactos culturales de orden tecnológico; $y$, finalmente, la constitución de una actitud crítica, reflexiva y comprensiva ante el mundo de la tecnología. Lo anterior es llevado a cabo por medio de talleres y seminarios que buscan generar conflictos y reelaboraciones a partir de lo que ya se sabe en confrontación y complementación con los nuevos aprendizajes.

En cuanto a la acción pedagógica, la experiencia anterior debe permitir la construcción de algunos conceptos para enfrentar la acción: pensamiento tecnológico y artefacto cultural y su implicación en el aula. 
En términos generales se habla de pensamiento tecnológico, en la medida que se propician espacios de vivencias de conocimiento (Segura, 1999: 33-41), es decir, en tanto el docente propone situaciones que enriquezcan el mundo de experiencias del niño, específicamente experiencias tecnológicas.

Las situaciones planteadas están enmarcadas en un contexto donde se generan necesidades o situaciones problema, en el cual, el niño pone en juego su creatividad e imaginación, diseñando y construyendo "posibles soluciones tecnológicas" a partir de sus propias ideas. Estas situaciones (que planea $y$ diseña el vivencialista $)^{10}$ deben ser desencadenantes, de tal forma que el niño tenga la necesidad de interactuar con los artefactos propuestos ${ }^{11}$. Esta interacción puede ser dada de dos maneras: en primera instancia, cuando se le lleva un artefacto en términos de desciframiento de cajas negras y agendas ocultas; $y$, en segundo lugar, cuando se le lleva otros artefactos ${ }^{12}$ con el fin de que el niño dé solución a una pregunta o tema planteado, construyendo otro artefacto.

\section{Metodología}

La intención fundamental de este proyecto de innovación en el aula universitaria, es poner en contacto a los estudiantes de la vivencia con la labor docente y darles las herramientas mínimas para llevar a cabo "un primer acercamiento al trabajo investigativo" en el aula escolar, a través de la implementación e interacción con artefactos, teniendo como conceptos relevantes el pensamiento tecnológico y el artefacto cultural. Para lograr dicho objetivo se partió de los resultados de la sistematización de la ficha cualitativa.

A fin de cumplir lo expuesto anteriormente, la metodología abordada para interpretar los datos que muestran las fichas cualitativas fue construida

10 Es el estudiante que está realizando la práctica docente o vivencia escolar.

11 El inventario de artefactos con el cual se cuenta está constituido por tuercas y tornillos, cubos, pentominó, engranaje, herramientas, lego, bloques lógicos, estructuras, armotodo, geofiguras y tangram (hasta el 2011-I).

12 Aquí se refiere a los artefactos que existen en el inventario de esta vivencia. como un proceso de sistematización de los mismos (datos ${ }^{13}$ ), visualizándose una mixtura entre lo cuantitativo y lo cualitativo, donde cada ficha tomada en esta sistematización representa el informe cualitativo de un niño.

\section{Avances del proceso}

La selección de las muestras de este universo, dada su cantidad, se llevó a cabo mediante un procedimiento aleatorio simple ${ }^{14}$. El total de las fichas de los semestres en estudio se presenta en el siguiente cuadro:

\begin{tabular}{|c|c|}
\hline SEMESTRE & No. FICHAS \\
\hline $2003-I$ & 22 \\
\hline $2003-$ II & 396 \\
\hline $2004-I$ & 210 \\
\hline $2004-$ II & 200 \\
\hline $2005-I$ & 647 \\
\hline $2005-$ II & 672 \\
\hline $2006-I$ & 602 \\
\hline $2006-$ II & 350 \\
\hline Total & $\underline{\mathbf{3 0 9 9}}$ \\
\hline
\end{tabular}

\section{Base de datos}

Para levantar la base de datos a partir de la información ofrecida tanto por las fichas como por nuestro soporte teórico (pensar tecnológicamente y artefacto cultural), se construyeron cuatro dimensiones: social, cognitiva, tecnológica e innovación ${ }^{15}$, con unas categorías para su respectiva lectura.

A partir de lo expuesto anteriormente y de la interpretación de dichos datos, se propuso una ficha cualitativa que diera cuenta puntualmente tanto del quehacer del niño con los artefactos, de su

13 Fichas cualitativas correspondientes a los semestres 2003 - I hasta el 2006 - II.

14 Para este proyecto de innovación, tomamos una muestra de la población finita (3.099), de tal forma que se garantizó a cada uno de los elementos de la población la misma oportunidad de ser incluidos en dicha muestra (232).

15 Espacio escuela-jardín y artefactos. 
interacción social (pares y adultos), como de los avances en los aspectos cognitivos para el favorecimiento del desarrollo del pensamiento tecnológico.

Estas dimensiones son:

Dimensión social: se refiere a aquellos aspectos donde el niño interactúa de forma directa tanto con sus pares como con los adultos, en este caso, estudiantes practicantes y profesores titulares de las instituciones.

Para abordar esta dimensión se diseñaron cuatro categorías que permitieron evidenciar las interacciones del niño, en las diferentes sesiones llevadas a cabo.
A: Trabajo individual
B: Trabajo colaborativo (ayuda)
C: Compartir material
D: Diálogo (niño con pares)

Dimensión cognitiva: representa las operaciones mentales y habilidades motrices (motricidad fina) que el niño debe o debería llevar a cabo, tanto para interactuar con el artefacto (encajando, manipulando, empatando, atornillando y moldeando), como para dar posibles soluciones al problema planteado o desarrollar el tema propuesto.

A: Clasificación (forma, color, tamaño y textura)

B: Concentración (MC: muy concentrado, tiempo mayor a $20 \mathrm{~min}$.)

(MDC: medianamente concentrado, un tiempo de 10 a 20 min.)

(PC: poco concentrado, un tiempo de 1 a $10 \mathrm{~min}$.) y

(NC: no concentrado, 0 minutos)

C: Encajar

D: Empatar

E: Atornillar

F: Moldear

G: Ubicación (lugar donde ubica las fichas de los artefactos) (Sí: ubica; NO: no ubica)
Dimensión tecnológica: describe las diferentes formas de asumir o abordar un problema o una temática. Estas formas abarcan desde una exploración del artefacto hasta una planeación con su respectivo diseño para llegar a la construcción de la probable solución del problema que, finalmente, es otro artefacto.

Lo anterior implica que los niños tanto para la situación problema como para el tema, plantean una solución tecnológica que se expresa en términos de un diseño y construcción de un nuevo artefacto.
A: Exploración del artefacto
B: Propuesta de solución
C: Planeación
D: Diseño (No elaborado: de 0 a 3 formas no inte- gradas; medianamente elaborado: de 4 a 8 formas integradas; elaborado: de 9 en adelante formas inte- gradas).
E: Implementación
F: Argumentación del niño
G: Diálogo del adulto con el niño

Dimensión Innovación: esta dimensión se refiere a los espacios (escuela y jardín) donde se trabajó hasta el año 2006 - II, y a los artefactos con los cuales fue posible llevar a cabo dicho trabajo.

\begin{tabular}{|l|l|l|}
\hline $\begin{array}{l}\text { MM: María Mon- } \\
\text { tessori }\end{array}$ & $\begin{array}{l}\text { 1: Artefacto ge- } \\
\text { neral }\end{array}$ & 6: Lego (perdido) \\
\hline DT: Diana Turbay & $\begin{array}{l}\text { 2: Tuercas y Tor- } \\
\text { nillos }\end{array}$ & 7: Estructuras \\
\hline $\begin{array}{l}\text { BI: Bosque Iz- } \\
\text { quierdo }\end{array}$ & 3: Pentominó & $\begin{array}{l}\text { 8: Bloques Ló- } \\
\text { gicos }\end{array}$ \\
\hline $\begin{array}{l}\text { MD: Minuto de } \\
\text { Dios }\end{array}$ & 4: Engranaje & $\begin{array}{l}\text { 9: Armatodo } \\
\text { (morrales) }\end{array}$ \\
\hline N: Nazareth & 5: Herramientas & $\begin{array}{l}\text { 10: Cubos y } \\
\text { palos }\end{array}$ \\
\hline
\end{tabular}

\section{Conclusiones}

Las conclusiones que a continuación se citan están referidas fundamentalmente a los aspectos que tienen que ver con la construcción, desarrollo e implementación de la ficha cualitativa, teniendo en 
cuenta el plan de trabajo (ver anexo 1), es decir, los insumos para la sesión de aula y la elaboración de la ficha cualitativa (ver anexo 2). También se encuentran algunas conclusiones referentes a la actitud del estudiante universitario y de la vivencia como tal.

- Es necesario insistir a los estudiantes en la forma cómo ellos plantean la situación problema $^{16}$, pues ésta, además de mediar entre el niño y los artefactos, debe ser acorde a las posibilidades de los mismos (artefacto). En otras palabras, el componente creativo para trabajar con niños de estas edades ${ }^{17}$ es muy importante.

- Se encontró que la mayoría de los niños argumentan e implementan la solución del problema. Esta situación puede ser enriquecida con el uso de preguntas desencadenadoras, que sustituyan preguntas como el por qué... induciendo una respuesta afirmativa o negativa, carente de argumento. Esto muestra la pertinencia de enfatizar en los estudiantes sobre la forma de preguntar.

- En lo referente a la dimensiones de la ficha cualitativa se encontró que en la tecnológica, las categorías construidas como el empatar, encajar y textura para leer las muestras, no fueron pertinentes ya que se presenta confusión entre encajar y empatar; además la textura, no pudo ser "vista" en las fichas, porque los artefactos con los que se cuenta no ofrecen gamas de estas ${ }^{18}$ (texturas). Las categorías moldear y ubicación, se prestaron para confusión en el momento de identificarlas en las muestras estudiadas. En el caso de moldear, no hay ningún artefacto del inventario que permita esta opción; y para ubicación, que es independiente del artefacto, no hay un refe-

16 Situación problema: muestra las propuestas de acercamiento al niño con el artefacto que hacen los estudiantes, para que él (niño) se disponga, se apropie, se interese, se motive a interactuar con éstos (artefactos). Este acercamiento se ha realizado a través de la propuesta de una situación problema (tema o pregunta) acorde a la edad del niño. Cuando se propone una situación problema, se crea un ambiente (cuento, narración, obra, títeres, película, etc.) y se concluye con una pregunta específica (¿Qué...? y ¿Cómo...?), cuya respuesta va encaminada a ayudar a los personajes míticos involucrados en la pregunta.

17 Niños de 4 a 6 años promedio.

18 Son solo de madera y de plástico. rente claro, por consiguiente se presentaron problemas de interpretación.

- Cuando se estableció la categoría de diseño, se hizo en términos de formas integradas y relacionadas con la solución del problema, pero no se hizo énfasis en el diseño de éstas, es decir, si son circulares, lineales, etc. Sin embargo, se encontró que el diseño (en formas) se presenta en todas las muestras estudiadas. Es válido aclarar que los estudiantes están pendientes de inducir en los niños este elemento (el diseño) como parte de un pensar tecnológicamente.

- Las dimensiones tecnológica, cognitiva y social son parte sustancial para visualizar el favorecimiento del pensamiento tecnológico. En el mismo sentido se sigue dando una retroalimentación por parte de los actores involucrados (profesores y estudiantes), en términos de especificar más claramente sus categorías.

- En el momento de la clasificación tanto de las fichas cualitativas como de los planes de trabajo se observó que, en estos últimos, se requería hacer más énfasis en la presentación de los datos generales de la misma; por esta razón, como parte de uno de los resultados de este proyecto de innovación en el aula ${ }^{19}$, se propuso un nuevo modelo para el plan de trabajo de esta vivencia (ver anexo 1).

- La elaboración de los registros ${ }^{20}$ el mismo día, asegura una información más objetiva y precisa. Este registro es una tarea dispendiosa pero relevante para visualizar el proceso tanto de los niños como de los estudiantes. El avance de la estructura de la ficha es significativo, pero se requiere de aportes sistemáticos para detallar las categorías y para que exista más apropiación de ésta por parte de los estudiantes. La ficha cualitativa no es un instrumento de evaluación, sino de descripción que posibilita visualizar, a través de sus dimensiones, el proceso del favorecimiento del pensamiento tecnológico del niño.

19 Formación pedagógica para favorecer el pensamiento tecnológico de niños y niñas de 4 a 6 años promedio.

20 Ficha cualitativa. 
- Es necesario, durante las horas teóricas de la vivencia (seminario de vivencia), aclarar los diferentes ítems o categorías de las dimensiones propuestas en la ficha cualitativa, con el fin de ver más allá de lo evidente cuando se está trabajando con los niños. Así, por ejemplo, el profesor debe tener la capacidad de hacer que los estudiantes apropien las técnicas de registro de información, pues esto es básico para el registro de la ficha cualitativa.

- Para el trabajo con los niños, en torno al favorecimiento del pensamiento tecnológico, se hace necesario que los estudiantes sean curiosos, adquieran otra mirada del mundo de la tecnología, tengan clara la presentación del problema para que los niños lo comprendan y puedan proponer soluciones "tecnológicas" al mismo. Es aquí donde lo propuesto por Michael Cole, "el artefacto cultural", contextualizado en este proyecto, adquiere sentido, ya que se trata de llevar al niño al mundo de la tecnología con los artefactos mediados por la situación problema.

- Esta vivencia, por su estado "en construcción" (aún en el 2011), permite al estudiante tomar un papel activo en ella. Aquí, él debe ser propositivo continuamente, de manera tal que no está dicha la última palabra para propiciar un ambiente que favorezca el pensar tecnológicamente. En consecuencia, su formación como docente está en un continúo enriquecimiento.

- Esta forma de trabajo, es exclusiva de la práctica formativa de cuarto semestre, y en los espacios en los cuales se ha interactuado, ninguna otra universidad (que tenga una carrera afín) ha tenido este tipo de acercamiento y de mirada: "favorecimiento del pensamiento tecnológico". Lo anterior conlleva a decir que esta vivencia escolar es un proyecto de innovación en el aula de la universidad.

\section{Bibliografía}

Becco, G. "Vygotsky y teorías sobre el aprendizaje: Conceptos centrales de la perspectiva vygotskyana", [en línea]. http://www.robertexto. com/archivo13/vygotsky.htm [consultado en junio de 2011].

Benlloch, M. (1992). Ciencias en el parvulario. España: Paidós.

Bruner, J. (1998). Realidad mental y mundos posibles. Barcelona: Gedisa.

Buch, T. (1999). Sistemas Tecnológicos: Contribuciones a una Teoría General de la Artificialidad. Argentina: Aique.

Cole, M. (1999). Psicología cultural: una disciplina del pasado y del futuro. España: Morata.

Edwards, D. \& Mercer, N. (1988). El conocimiento compartido: el desarrollo de la comprensión en el aula. España: Paidós.

Fourez, G. (1994). Alfabetización Científica y Tecnológica: acerca de las finalidades de la enseñanza de las ciencias. Argentina: Colihue.

Kuhn, T. (1974). La estructura de las revoluciones científicas. México: Fondo de Cultura Económica.

Mojica, L. (2003). En trabajos inéditos: el aprendizaje un acontecimiento mediado por agregados de artefactos. [En CD-ROM]. Colombia: INTERCITEC, Universidad Distrital.

Perkins, D. (1989). Conocimiento como diseño. Bogotá: Universidad Javeriana.

Segura, D. (1999). La construcción de la confianza: una experiencia de proyectos de aula. Colombia: Corporación Escuela Pedagógica Experimental.

Torres, O., Pedreros, R., et al. (1999). La autorregulación: un universo de posibilidades. Colombia: Fuego Azul. 
Anexo 1. Plan de trabajo

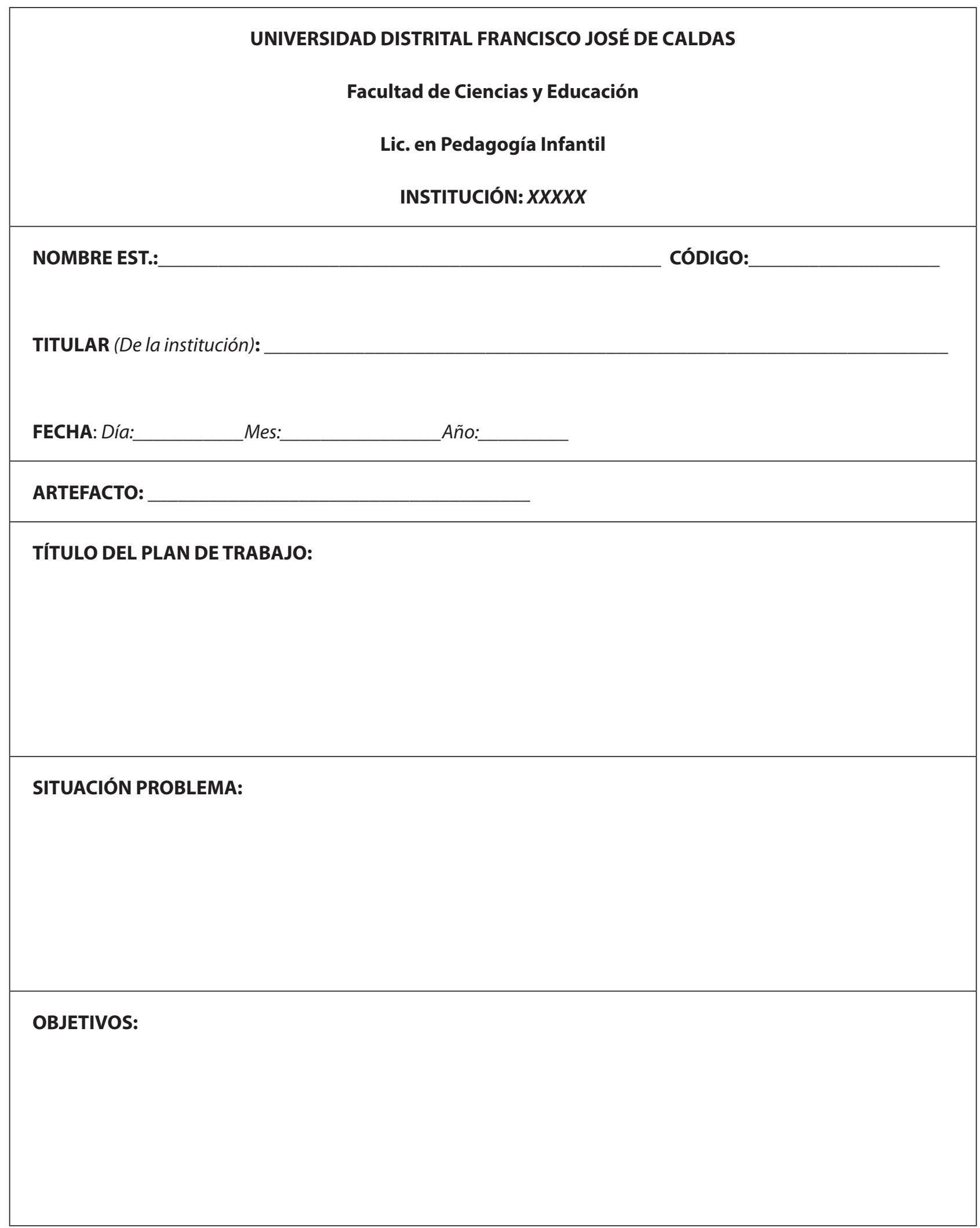


METODOLOGÍA (tiempos):

RECURSOS (artefacto y materiales anexos):

OBSERVACIONES: 


\section{Anexo 2. Ficha cualitativa}

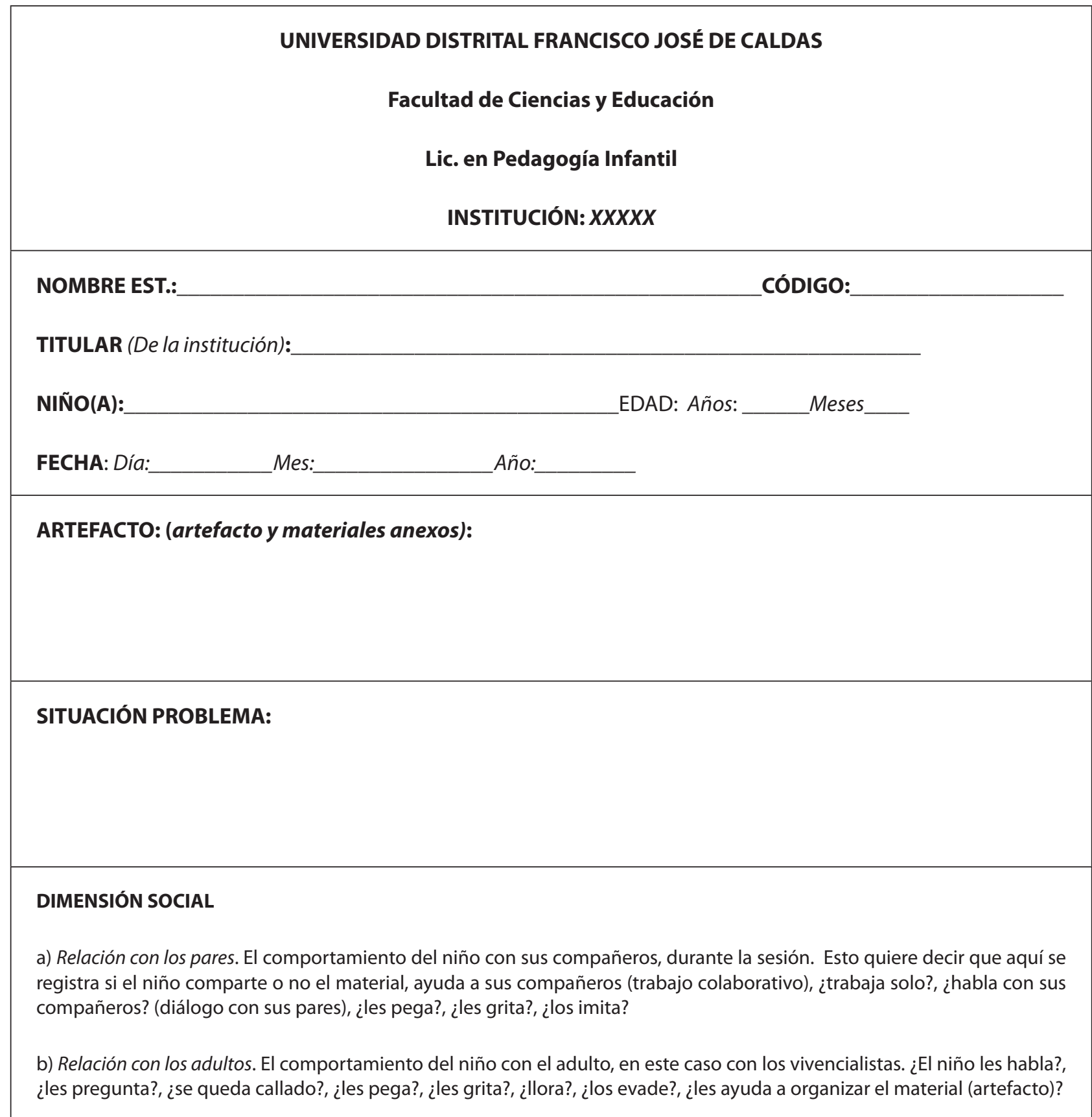




\section{DIMENSIÓN TECNOLÓGICA}

a) Actitud frente al problema. ¿Cómo el niño comprendió la situación problema? ¿El niño se presenta disperso frente al planteamiento del problema? ¿El niño se muestra atento frente al problema?

b) Actitud frente al artefacto. ¿El niño explora y manipula el artefacto (interacciona)? ¿Pregunta sobre el uso o utilidad de las fichas que componen el artefacto?

c) Planeación. ¿Expresa de manera clara y rápida la posible solución al problema? ¿Expresa un procedimiento (por ejemplo, una lista de actividades) para dar solución al problema?

d) Diseño' ¿ ¿El niño plasma su posible solución?, ¿la aurgumenta?² Se le pide o induce al niño para que dibuje su posible solución. En este caso, se establecen unos criterios de "dibujo elaborado", acorde con el número de formas o figuras que utilice en su diseño, las cuales deben estar relacionadas con la solución del problema (No elaborado: cero o formas no integradas; medianamente elaborado: de 1 a 5 formas integradas; elaborado: de 6 en adelante formas integradas). Sus trazos (en el dibujo), ¿son líneas o curvas? ¿Éstos son definidos?

e) Construcción (implementación). Aquí se describe, paso a paso, la solución que el niño dio al problema con el uso del artefacto. Se mira si existe coherencia entre lo diseñado con lo implementado. Aquí se debe establecer también una relación dialógica con el niño para entender su solución y el por qué de lo que hizo (diálogo adulto - niño).

d) Explicaciones construidas, relacionadas con la solución o construcción. En este ítem se retoman las explicaciones y argumentaciones del anterior.

\section{DIMENSIÓN COGNITIVA}

Clasificación: esta operación permite distinguir de forma detallada las diferentes características del artefacto. Para este caso, el niño organiza los elementos de éste por forma, color y/o tamaño.

Concentración: con esta categoría se quiere ver el tiempo que, en el proceso de interacción con el artefacto, el niño logra dedicar exclusivamente a ésta. A continuación se muestra una serie de rangos o intervalos que el equipo de investigación determinó, de acuerdo con la experiencia de aula: (MC: muy concentrado, tiempo mayor a 20 min.), (MDC: medianamente concentrado, un tiempo de 10 a 20 min.), (PC: poco concentrado, un tiempo de 1 a 10 min.) y (NC: no concentrado, 0 minutos).

Encajar: se refiere a los diferentes elementos del artefacto, meter uno dentro de otro, de manera que quede ajustado.

Empatar: empalmar o unir dos o más elementos de un artefacto.

Atornillar: este caso se ve desde dos puntos de vista: uno corresponde al tornillo mismo, que sería introduciéndolo en una superficie, haciéndolo girar alrededor de su eje; y el segundo, cómo el niño logra sujetar por medio de tornillos dos partes.

Ubicación: es la correspondencia entre lo representado bidimensionalmente (plano, esquema, dibujo) con la representación tridimensional (artefacto "maqueta" construido con artefactos).

\section{OBSERVACIONES:}

1 En este ítem, en la medida de lo posible, solicitar al niño su diseño en términos gráficos. Lo cual implica que debe llevarse hojas para que pueda dibujar. Todos los resultados de la sesión se anexan a esta ficha.

2 Recordar que, a partir del diseño, hay que establecer una relación dialógica. 